\title{
BMJ Open Resource utilisation and costs in predementia and dementia: a systematic review protocol
}

Filipa Landeiro, ${ }^{1}$ Helena Wace, ${ }^{1}$ Isaac Ghinai, ${ }^{1}$ Elsbeth Nye, ${ }^{1}$ Seher Mughal, ${ }^{1}$ Katie Walsh, ${ }^{1}$ Nia Roberts, ${ }^{2}$ Pascal Lecomte, ${ }^{3}$ Raphael Wittenberg, ${ }^{4}$ Jane Wolstenholme, ${ }^{1}$ Ron Handels, ${ }^{5,6}$ Emilse Roncancio-Diaz, ${ }^{7}$ Michele H Potashman, ${ }^{8}$ Antje Tockhorn-Heidenreich, ${ }^{9}$ Alastair M Gray, ${ }^{1}$ on behalf of the ROADMAP Group

To cite: Landeiro $F$, Wace $\mathrm{H}$, Ghinai I, et al. Resource utilisation and costs in predementia and dementia: a systematic review protocol. BMJ Open 2018;8:e019060. doi:10.1136/ bmjopen-2017-019060

- Prepublication history and additional material for this paper are available online. To view these files, please visit the journal online (http://dx.doi. org/10.1136/bmjopen-2017019060).

Received 8 August 2017 Revised 10 November 2017 Accepted 13 November 2017
Check for updates

For numbered affiliations see end of article.

Correspondence to

Filipa Landeiro;

filipa.landeiro@dph.ox.ac.uk

\section{ABSTRACT}

Introduction Dementia is the fastest growing major cause of disability globally with a mounting social and financial impact for patients and their families but also to health and social care systems. This review aims to systematically synthesise evidence on the utilisation of resources and costs incurred by patients and their caregivers and by health and social care services across the full spectrum of dementia, from its preceding preclinical stage to end of life. The main drivers of resources used and costs will also be identified.

Methods and analysis A systematic literature review was conducted in MEDLINE, EMBASE, CDSR, CENTRAL, DARE, EconLit, CEA Registry, TRIP, NHS EED, SCI, RePEC and OpenGrey between January 2000 and beginning of May 2017. Two reviewers will independently assess each study for inclusion and disagreements will be resolved by a third reviewer. Data will be extracted using a predefined data extraction form following best practice. Study quality will be assessed with the Effective Public Health Practice Project quality assessment tool. The reporting of costing methodology will be assessed using the British Medical Journal checklist. A narrative synthesis of all studies will be presented for resources used and costs incurred, by level of disease severity when available. If feasible, the data will be synthesised using appropriate statistical techniques.

Ethics and dissemination Included articles will be reviewed for an ethics statement. The findings of the review will be disseminated in a related peer-reviewed journal and presented at conferences. They will also contribute to the work developed in the Real World Outcomes across the Alzheimer's disease spectrum for better care: multi-modal data access platform (ROADMAP). Trial registration number CRD42017071413.

\section{INTRODUCTION}

Dementia is the fastest growing major cause of disability globally. ${ }^{1}$ According to the World Alzheimer Report 2016, over 47 million people live with dementia worldwide. This number is predicted to almost double every 20 years with over 130 million expected to live
Strengths and limitations of this study

- This systematic literature review on resource use and costs of dementia was based on a detailed search strategy.

- Search strategy did not specify countries of interest or limit languages.

- The review follows robust guidelines and the quality of the papers included will be assessed using a validated tool

- The heterogeneity in the reported measures of resource use and costs may not enable direct comparisons between studies to be made.

with the condition by $2050^{2}$. The human and financial burden associated with this disease is vast: the global cost was estimated to be US $\$ 818$ billion in 2015 , equivalent to more than the Gross Domestic Product (GDP) of countries such as Switzerland and the Netherlands. ${ }^{3}$ Dementia is predicted to become a trillion dollar disease by $2018^{4}$. It therefore represents a very relevant challenge from both a medical and economic perspective.

Dementia is a debilitating condition for which there is no cure. It is a progressive neurodegenerative syndrome characterised by cognitive, behavioural and neuropsychiatric changes which impair social function and activities of daily living (ADLs). Alzheimer's disease $(\mathrm{AD})$ is the most common cause of dementia, accounting for one half to three quarters of all cases. ${ }^{5}$ Other forms of dementia include vascular dementia, dementia with Lewy bodies and frontotemporal dementia among others. There is increasing recognition of co-occurrence of different forms of dementia-especially vascular pathologies alongside $\mathrm{AD}$. What all the diseases share in common is an inevitable decline in cognition, such that patients are in need of extensive 
personal care, including supervision and assistance with ADLs.

The financial cost of dementia is vast and continually growing but remains difficult to quantify. In the last decade, a few reviews have analysed the cost of illness data in dementia, with the last update conducted in $2013{ }^{6-8}$ These have highlighted the significant direct non-medical costs associated with care, primarily informal care costs and costs of formal services both home-based and in care homes in addition to the direct medical costs. These reviews have also drawn attention to the indirect costs which arise from the effect on families, carers and the community. These are more challenging to measure and there is significant variation in the methodology used to estimate these costs. Many of these recent reviews were limited to studies addressing $\mathrm{AD}$ alone and they did not include studies reported in languages other than English. Furthermore, these reviews did not quantify the costs associated with predementia states including preclinical AD (biomarker positive but presymptomatic), early symptoms, mild cognitive impairment (MCI) or prodromal AD. Given the constantly increasing number of dementia patients, further investigation is warranted to analyse the factors which are driving the soaring costs. This review aims to address the following questions:

i. What type and level of resources are used by patients with dementia in all stages of dementia disease severity, from preclinical to end of life, and their caregivers?

ii. What are the direct, indirect and informal costs of these patients and caregivers?

iii. Are resource use and costs significantly associated with specific factors?

\section{METHODS AND ANALYSIS \\ Protocol and registration}

This protocol was prepared according to the reporting guidelines of the Preferred Reporting Items for Systematic Reviews and Meta-Analysis for Protocols 2015 (PRISMA-P) ${ }^{9}$ and the completed PRISMA-P checklist can be found in the online supplementary file 1 . The review protocol was registered with the PROSPERO international prospective register of systematic reviews (registration number CRD42017071413). The systematic review manuscript will be prepared following the PRISMA statement. ${ }^{10-12}$ Important amendments to this protocol will be reported and published with the results of the review.

\section{Study selection criteria \\ Participants}

Studies will be included if the full or part of the study population is reported to have either preclinical (presymptomatic) $\mathrm{AD}$; early symptoms; $\mathrm{MCI}$; prodromal $\mathrm{AD}$; mild, moderate or severe dementia or is a caregiver of a person with predementia or dementia, irrespective of the type and stage of the disease. However, only studies focusing on adult populations will be considered for inclusion.

\section{Study design}

This systematic review will include studies published in peer-reviewed journals or gray literature, where outcomes were estimated based on patient-level data. In terms of study design, both interventional and observational studies will be considered for inclusion (regardless of whether a prospective or retrospective approach is used) as well as register-based studies. Where multiple studies used the same patient cohort, data extraction will be restricted to the study that presented the most detailed information on resources used and costs for dementia. Furthermore, case studies, series of case studies, studies with sample size of $<30$ patients, trial protocols, phase I clinical trial, news articles, interviews (that do not use a structured quantitative questionnaire), patient education handouts, reviews, opinion or expert articles, editorial, letters to the editor, authors and editor's replies to comments will not be included.

Modelling studies will be excluded but studies informing the model parameters will be considered for inclusion. Similarly, reviews of existing economic studies on dementia will not be included but their reference lists will be screened for additional studies. Conference abstracts will be screened in order to determine whether the work presented had been published in a peer-reviewed journal or gray literature. However, the abstracts themselves will not be considered for inclusion.

\section{Outcomes}

The outcomes of interest are as follows:

- The resources used by patients and their caregivers.

- The costs incurred by patients and their caregivers.

Where possible, both resources used and costs incurred will be detailed by stage of the disease: preclinical $\mathrm{AD}$ (biomarker positive but presymptomatic), early symptoms (memory and other behavioural changes), MCI, prodromal $\mathrm{AD}$, mild dementia, moderate dementia, severe dementia and end of life in order to understand how costs evolve throughout disease progression. Data on any further subdivision of stage of disease will also be extracted if described in studies.

Where studies present multiple data points, all relevant data points will be individually extracted and kept separate for analysis. For example, data will be individually extracted from studies that present data from multiple time points, studies that present data from multiple countries and studies that present data from multiple settings (eg, community and institutionalised).

The costs will be presented as annual costs incurred per patient, and will be linearly extrapolated or interpolated if necessary. Costs will be extracted in local currency. However, if the cost estimates are not reported in local currency, these will be converted to local currency using the annual reported currency exchange rates for the year of costing. All cost estimates will be inflated to the last 
available year with deflator data for all countries included in the review, using the World Bank GDP deflator for each country. ${ }^{13}$ Inflated costs will be converted to USD using the GDP Purchasing Power Parity conversion rates for each country. ${ }^{14}$

\section{Intervention}

All dementia-related interventions, either symptomatic or disease modifying, will be included. We will also consider studies that have not assessed any intervention.

\section{Language}

No language restrictions were applied to the search.

\section{Setting}

No geography or clinical setting restrictions were applied to the search.

\section{Search strategy}

\section{Electronic databases}

Electronic databases were selected in conjunction with an information specialist. The search terms were developed based on previous literature reviews' search strategies $^{15}$ with the assistance of the information specialist. The following electronic databases were searched from 1 January 2000 to 4 May 2017: Medical Literature Analysis and Retrieval System Online (MEDLINE), Excerpta Medica dataBASE (EMBASE), Cochrane Database of Systematic Reviews (CDSR), Central Register of Controlled Trials (CENTRAL), Database of Abstracts of Reviews of Effects (DARE), EconLit, Cost-Effectiveness Analysis Registry (CEA Registry), Turning Research Into Practice (TRIP), NHS Economic Evaluation Database (NHS EED), Science Citation Index (SCI), Research Papers in Economics (RePEc) and OpenGrey Repository (OpenGrey). The exact search terms used in all databases are described in the online supplementary file 2 . The database searches have now been completed.

\section{Manual searches}

The reference list of the studies included in this review, as well as those of previous literature reviews on resources used and costs across the full spectrum of dementia, will be searched in order to identify additional potentially relevant studies. The studies informing the model parameters in identified modelling studies will be considered for inclusion. Conference abstracts identified through the electronic searches will be screened and manuscripts of relevant abstracts will be manually searched for.

\section{Study selection}

The references will be managed in ENDNOTE X7, Thomson Reuters. The removal of duplicates in terms of title and first author name will be performed by one reviewer. Afterwards, two reviewers will independently assess the titles and abstract of the studies to determine whether full text review is needed. Disagreement between the two reviewers will be resolved by a third reviewer. Full text of potentially eligible studies will be retrieved and reviewed and assessed for final inclusion by two reviewers with a third reviewer being consulted in case of disagreement. Non-English references will be screened by native or fluent speaker identified from a pool of collaborators at the University of Oxford. A flow diagram will be created to illustrate the selection process according to PRISMA guidelines. ${ }^{10}$

\section{Data extraction}

Data will be independently extracted by two of following reviewers (EN, ERD, FL, IG, HW, RH, SM) using a data extraction form (online supplementary file 3) with disagreements being solved by the third reviewer. Non-English references will be reviewed by two native or fluent speakers. The following information will be extracted:

- Study details: title, author, publication details, location, language of the study, countries of the study.

- Study design: aim of the study, type of study, type of analysis, duration, outcomes measured, instruments used to measure them.

- Participant information: type of participant, setting, inclusion and exclusion criteria, sample size, sociodemographic information.

- Disease specific information: type of dementia, level of severity, instrument used to measure level of severity.

- Outcomes: outcomes measured, subgroup analysis conducted, adjustments carried out.

- Results: resources used by patients or their caregivers by disease severity, costs incurred by patients or their caregivers by disease severity, type of costs, costing methodology, cost perspective.

- Conclusions: authors' conclusions.

Risk of bias (quality) assessment

A quality assessment of each study will be independently conducted by two of following reviewers (EN, ERD, FL, IG, HW, RH, SM) using the Effective Public Health Practice Project 'Quality assessment tool for quantitative studies ${ }^{16}$ recommended by the Cochrane Public Health Group as it covers a wide range of study designs. ${ }^{17}$

A quality assessment for the reporting of the costing methodology will also be undertaken using a shortened version ${ }^{18}$ of the British Medical Journal Checklist to assess the quality of economic evaluations ${ }^{19}$ (online supplementary file 4$)$.

The quality assessment of non-English studies will be carried out by two native or fluent speakers.

\section{Description of studies and analysis}

We expect to find a diverse range of study designs and heterogeneous presentations of resources used and costs. Data will be presented in terms of annual cost per patient, as detailed above. Costs will be presented separately, when possible, for the different types of dementia, but with a special emphasis on $\mathrm{AD}$, the most common form of dementia. If possible, cost data will be presented for each stage of the disease. Costs will also be subdivided 
by cost category: direct (medical and non-medical) costs, indirect costs and informal costs. ${ }^{2021}$

A narrative synthesis of all relevant studies will be provided discussing differences in resources used and costs incurred by type of dementia, disease severity, type of cost, cost perspective, study design, and describing study and participants' characteristics, results and author's conclusions.

The authors of the studies included in this review will be contacted with the aim to retrieve any missing data necessary for our analysis.

\section{DISCUSSION}

This systematic review will synthesise the resources used and costs incurred by patients across the full spectrum of dementia from predementia through dementia to end of life. The resources used and costs incurred by their caregivers will also be summarised. Detailing the consumption of resources and associated costs throughout the different stages of the disease can contribute to a better understanding of the economic impact of dementia and also better inform disease progression and cost-effectiveness models, especially when in some cases costs were reported to be more than double between later stages of the disease. ${ }^{22}$

Even though our main interest is $\mathrm{AD}$, given the lack of diagnostic accuracy and the recognised overlap between different causes of dementia, all forms of dementia will be considered and the costs will be presented separately by disease type, if the information is available.

The quality of the studies included in this review will be assessed and, if feasible, the resource use and cost data will be synthesised using appropriate statistical methods. Furthermore, this review will include studies conducted in the last 17 years without any languages or geographic restrictions in contrast to previous reviews which were restricted to studies published in English language up to 2013. The review was limited to studies published in or after the year 2000 to ensure that the results of the review were applicable to present settings. Only one previous review $^{6}$ assessed the quality of the studies.

In conclusion, this review aims to address the economic impact of dementia, globally recognised as a health priority not only in terms of the impact it has on older people's health but also on health and social care systems worldwide. Understanding the economic implications of this disease will allow policy-makers to devise better health and social care plans for both patients with dementia and their caregivers.

\section{Author affiliations}

${ }^{1}$ Nuffield Department of Population Health, Health Economics Research Centre, University of Oxford, Oxford, UK

${ }^{2}$ Bodleian Health Care Libraries, University of Oxford, Oxford, UK

${ }^{3}$ Global Head Health Economic Modelling and Methodology, Novartis Pharmaceutical AG, Basel, Switzerland

${ }^{4}$ Personal Social Services Research Unit, London School of Economics and Political Science, London, UK
${ }^{5}$ Department of Psychiatry and Neuropsychology, Maastricht University, School for Mental Health and Neurosciences, Alzheimer Centre Limburg, Maastricht, The Netherlands

${ }^{6}$ Division of Neurogeriatrics, Department of Neurobiology, Care Science and Society, Karolinska Institute, Stockholm, Sweden

${ }^{7}$ GE Healthcare Life Sciences, Amersham, UK

${ }^{8}$ Value and Access, Biogen, Cambridge, Massachusetts, USA

${ }^{9}$ Eli Lilly and Company, Erl Wood Manor, Windlesham, UK

Acknowledgements The authors would like to acknowledge the contributions of the remaining members of Work Package 5 as well as of those of the wider ROADMAP group.

Collaborators Novartis Pharma AG, GE Healthcare, Biogen and Eli Lilly and Company Limited are industry partners in the ROADMAP Project.

Contributors FL: designed the study and is the guarantor of the review. FL, HW and KW: wrote the protocol. FL, NR, RW, JW, RH, MHP, ATH and AMG devised the search strategy. IG, EN, SM, PL, RW, JW, RH, ER-D, MHP, AT-H and AMG: critically appraised the protocol and also contributed to its development by revising different versions. All authors: read and approved the final version of the manuscript.

Funding This project has received funding from the Innovative Medicines Initiative 2 Joint Undertaking under grant agreement no 116020 (ROADMAP). This Joint Undertaking receives support from the European Union's Horizon 2020 research and innovation programme and EFPIA. RH has received consulting fees from Roche,Nutricia and Piramal; research grants from private/public collaborations:IMI2-ROADMAP; Alzheimer Netherlands Flutemetamol; CTMM LEARN Grant $02 \mathrm{~N}-101$; and public grants from: JPND Actifcare; and JPND BIOMARKAPD.

Competing interests This SLR is being conducted as part of the ROADMAP project. ROADMAP is a consortium of academic, public sector and industry partners working collaboratively within the European Union Innovative Medicines Initiative framework. FL, HW, IG, EN, SM, KW, NR, RW, JW and AMG declare that they have no competing interests. PL is employed by, owns stock in and has stock options in Novartis Pharma AG. ERD is employed by GE Healthcare. MP is an employee of Biogen and owns stock in Biogen. She owns stock in a variety of companies that at times include other pharmaceutical and health care-related companies. ATH is an employee of Eli Lilly and Company Limited and owns stock in Eli Lilly and Company Limited. Novartis Pharma AG, GE Healthcare, Biogen and Eli Lilly and Company Limited are industry partners in the ROADMAP Project.

Patient consent Not required.

Provenance and peer review Not commissioned; externally peer reviewed.

Open Access This is an Open Access article distributed in accordance with the Creative Commons Attribution Non Commercial (CC BY-NC 4.0) license, which permits others to distribute, remix, adapt, build upon this work non-commercially, and license their derivative works on different terms, provided the original work is properly cited and the use is non-commercial. See: http://creativecommons.org/ licenses/by-nc/4.0/

(C) Article author(s) (or their employer(s) unless otherwise stated in the text of the article) 2018. All rights reserved. No commercial use is permitted unless otherwise expressly granted.

\section{REFERENCES}

1. OECD. Addressing Dementia: OECD Publishing.

2. Prince M, Comas-Herrera A, Knapp M, et al. World Alzheimer report 2016: improving healthcare for people living with dementia: coverage quality and costs now and in the future, 2016.

3. Wimo A, Guerchet M, Ali GC, et al. The worldwide costs of dementia 2015 and comparisons with 2010. Alzheimers Dement 2017;13:1-7.

4. Prince MJ, Wimo A, Guerchet MM, et al. World Alzheimer Report 2015 - The Global Impact of Dementia: Alzheimer's Disease International, 2015.

5. World Health Organization. Neurological disorders: public health challenges: World Health Organization, 2006.

6. Colucci L, Bosco M, Fasanaro AM, et al. Alzheimer's disease costs: what we know and what we should take into account. J Alzheimers Dis 2014;42:1311-24.

7. Schaller S, Mauskopf J, Kriza C, et al. The main cost drivers in dementia: a systematic review. Int J Geriatr Psychiatry 2015;30:111-29. 
8. Takizawa C, Thompson PL, van Walsem A, et al. Epidemiological and economic burden of Alzheimer's disease: a systematic literature review of data across Europe and the United States of America. $J$ Alzheimers Dis 2015;43:1271-84.

9. Shamseer L, Moher D, Clarke M, et al. Preferred reporting items for systematic review and meta-analysis protocols (PRISMA-P) 2015: elaboration and explanation. BMJ 2015;349:g7647.

10. Moher D, Liberati A, Tetzlaff J, et al. Preferred reporting items for systematic reviews and meta-analyses: the PRISMA statement. PLoS Med 2009;6:e1000097.

11. Liberati A, Altman DG, Tetzlaff J, et al. The PRISMA statement for reporting systematic reviews and meta-analyses of studies that evaluate health care interventions: explanation and elaboration. PLoS Med 2009;6:e1000100.

12. Welch V, Petticrew M, Tugwell P, et al. PRISMA-Equity 2012 extension: reporting guidelines for systematic reviews with a focus on health equity. PLoS Med 2012;9:60-7.

13. The World Bank. GDP deflator (base year varies by country). Secondary GDP deflator (base year varies by country). http://data. worldbank.org/indicator/NY.GDP.DEFL.ZS

14. Organisation for Economic Co-operation and Development. Conversion rates - purchasing power parities. Secondary conversion rates - purchasing power parities 2017. https://data.oecd.org/ conversion/purchasing-power-parities-ppp.htm
15. Bond M, Rogers G, Peters J, et al. The effectiveness and costeffectiveness of donepezil, galantamine, rivastigmine and memantine for the treatment of Alzheimer's disease (review of Technology Appraisal No. 111): a systematic review and economic model. Health Technol Assess 2012;16:1-470.

16. Thomas H. Quality assessment tool for quantitative studies. Toronto: Effective Public Health Practice Project McMaster University, 2003.

17. Armijo-Olivo S, Stiles CR, Hagen NA, et al. Assessment of study quality for systematic reviews: a comparison of the Cochrane Collaboration Risk of Bias Tool and the Effective Public Health Practice Project Quality Assessment Tool: methodological research. $J$ Eval Clin Pract 2012;18:12-18.

18. Luengo-Fernandez R, Gray AM, Rothwell PM. Costs of stroke using patient-level data: a critical review of the literature. Stroke 2009;40:e18-23.

19. Drummond MF, Jefferson TO. Guidelines for authors and peer reviewers of economic submissions to the BMJ. BMJ 1996;313:275-83.

20. Jo C. Cost-of-illness studies: concepts, scopes, and methods. Clin Mol Hepatol 2014;20:327-37.

21. World Health Organization. The world health report: health systems financing: the path to universal coverage. World health report. 2010.

22. Quentin W, Riedel-Heller SG, Luppa M, et al. Cost-of-illness studies of dementia: a systematic review focusing on stage dependency of costs. Acta Psychiatr Scand 2010;121:243-59. 